\title{
Dark Organizational Theory
}

\section{Steven D. Brown, University of Leicester}

Paula Reavey, London South Bank University

\begin{abstract}
Institutions and organizations are defined by competing sociomaterial logics. Divergence between the 'visible' and the 'hidden' side of organization invites a critical work of 'unveiling'. But such critique does not enable understanding of how coherency is accomplished between different modes of reason. This is performed in emergent third spaces, where parasitic relations are enacted. During moments of 'crisis' or 'breach', contradictions are both acknowledged and given concrescence. Management comes into being in the anticipation of its breaking. Four accounts of this process are offered - a discussion of a remark from Michel Serres's The Parasite, a description of China Miélville's novel The City and The City, stories from fieldwork in medium-secure forensic psychiatric units, and set of conceptual propositions. Together they perform a descriptive practice called 'dark organization theory' which analyses the functional aspects of divergence and breaking in management and organizational practices.
\end{abstract}

\section{Preface}

It is an established truism to point to the competing 'sociomaterial logics', or specific local modes of constituting meaning through shaping and ordering diverse discourses and materials, that define organizations and institutions. There are numerous agendas and programmes of action that rub up against each other in complex and often contradictory ways. This much we know from Organization Studies (Cooper, 2016; Knox et al, 2015), Science \& Technology Studies (Mol, 2008; Mol \& Law, 2002) and Institutional Theory (Friedland \& Alford, 1991; Thornton et al, 2012). Naming these tensions can proceed in many different ways: formal and informal organization; espoused logic and theoriesin-use; low-level discourse and higher-level belief; systems and networks; positions and relations and so on. This dependency can also been framed in terms of 'light' and 'darkness' (see Burrell, 1997). Linstead et al, (2014) argue that negative or problematic behaviour on the part of organizational members and, indeed, the organization as whole, should not be seen as driven by external forces but rather as a constitutive 'inside'. The neo-psychoanalytic 'dark side' or unacknowledged, unconscious aspect of organization manifests itself in terms of misbehavior, destruction, violence and oppression. This invites a critical labour of demonstrating how the dark side is enacted as a hidden hand within the overt work of organization (see Hanlon, 2015).

The divergence and contrast between competing logics should not, however, lead us towards a notion of the visible and the hidden ${ }^{1}$. Organizations 'function' or 'work' in spite of, of perhaps because of, their inherently contradictory composition. There is no mystery to be unveiled here. We do not challenge management by pointing to a dark side that it scarcely takes the trouble to hide in the first place. But considering the coherence, or co-existence, of different logics raises the problem of how they intersect. At what point does one order of 
reason transform into another? Where is the moment of switching or reversibility? Could this point, this space, be treated as a 'third' that emerges between any two (or more) given orders? What status could it be accorded? And in what sense would it be implicated in the management of incommensurability? These kinds of questions cannot really be approached 'head on', since to do so risks prematurely lapsing into one order of reason to the exclusion of others. If the 'third space' is emergent, if it denotes a provisional and elusive moment between the two (or more) visible forms of order, then we need to stage a performance where that emergence takes place. This essay consists of four individual pieces that perform the opening of thirdness through different means: philosophical, literary, empirical and conceptual ${ }^{2}$. Each individual piece gnaws at the same problem - the emergent gap or 'breach' that erupts between competing logics. Taken together they suggest a way of approaching organizational discontinuities in a sideways fashion, glancing out of the corner of our eyes.

\section{I}

The point of departure is with a remark that Michel Serres makes towards the start of The Parasite. The book opens by narrating La Fontaine's version of the parable of the City Rat and the Country Rat. The story goes that the country cousin visits his relative in the city. At night, they creep out onto a kitchen table filled with the spoils of a feast. Both begin to take their fill until they are suddenly disturbed by a noise at the door and, taking fright, scurry away into the darkness. Serres offers a small diagram to illustrate the chain of actions involved in the story:

<insert Figure 1 here>

(Fig. 1 After Serres, 1982: 4)

The diagram indicates a succession or chain of thefts or parasitisms. The food on the table comes from somewhere, it is the booty of an initial theft left laying on the table. The city rat 'intercepts' this in a second act of parasitism, which becomes itself the basis for a further act of theft by the cousin. And finally there is another interruption. One more parasite is waiting at the door to take their place at the purloined feast.

Serres then goes on to remark:

Look again at the diagram based on the story of the rats, paying attention to the succession of parasites in a stepladder formation, and ask yourself if something is added to the system, like a cancer of interceptions, flights, losses, holes, trapdoors - if it is a pathological growth in some spot or if it is quite simply the system itself. The rats climb onto the rug when the guests are not looking, when the lights are out, when the party's over. Its nighttime, black. What happens would the obscure opposite of conscious and clear organization, happening behind everyone's back, the dark side of the system. But what do we call these nocturnal processes? Are they destructive or constructive? What happens at night on the rug covered with crumbs? Is it a still active trace of (an) origin? Or it is only a remainder of failed suppressions? We can, undoubtedly, decide the 
matter: the battle against rats is already lost; there is no house, ship, or palace that does not have its share. There is no system without parasites. This constant is a law. But how so? (Serres, 1982: 12, emphasis added)

The nighttime activities of the rats, the parasites, constitute, for Serres, a 'dark side of the system'. How to understand this phrase? We can start by rejecting a series of alternatives. Serres clearly does not locate the action of the rats as emanating from a source that is external to the system. This is no exogenous shock. The rats are not outsiders - they are internal to the very functioning of the system. Hence 'there is no system without parasites'. Moreover, the 'dark side of the system' is not some hidden aspect, a topographical 'underworld' or 'unconscious' that is suppressed from its normative functioning. Recently this notion of uncovering 'the dark side of organization' has taken hold in Management and Organization Studies (Raufflet \& Mills, 2009; Tourish, 2013). Work in the area aims to bring to light 'unseen' or 'dirty' work (e.g. Ward \& McMurry, 2015), or hidden and secret histories of organizations and their practices (Hanlon, 2015). But often it seems the only people who were actually unaware of these 'dark sides' were organization analysts. They were perfectly visible to those who had to routinely engage with them. All organizations engage to some degree in banal violence, humiliation and degradation in an entirely open fashion alongside their apparent formal good practices. This is not what Serres means by a 'dark side of the system'.

We need to think instead of the interdependency of the different aspect of the system with one another. The 'dark side of the system' could be thought of as that which enables, allows for, perhaps even calls for, the constitution of the apparent order of 'conscious and clear organization'. This is the position that Bruno Latour works out in detail from We Have Never Been Modern onwards. Substituting the terms 'purification' and 'hybridization' for light and dark, Latour famously argues for two generalized modes of ordering being conducted in concert with one another. The ongoing work of producing dualisms brings with it an increased entanglement of relations. There are no systems that are not, at the same time, networks. There are no pure terms without the thousands of mediators through which they are accomplished. The problem is the denial of this practical reality in formal epistemology:

The moderns have always been using both dimensions in practice, they have always been explicit about each of them, but they have never been explicit about the relation between the two sets of practices. Nonmoderns have to stress the relations between them if they are to understand both the moderns' successes and their recent failure, and still not lapse into postmodernism. By deploying both dimensions at once, we may be able to accommodate the hybrids and give them a place, a name, a home, a philosophy, an ontology, and, I hope, a new constitution. (Latour, 1993: 51)

The gesture of 'deploying both dimensions' at once is auto-deconstructive. Merely placing a sketch of the network in the same space of that as the system demonstrates both the necessity and the impossibility of properly thinking their interdependency. The organization is hierarchies, goals, sub-systems and 
functions. The organization is rhizomes, interceptions, quasi-objects and translations. A diagram of these two contradictory descriptions juxtaposed together invites a creative work of narrating the point where one slides into the other. Typically this takes the form of positing one dimension as the eventual limit of the other. Dualism, on this account, is what a network looks like in its most distended form, whilst translation becomes the mythic originary matrix in which opposed terms find their point of co-incidence and 'originary' coemergence.

<insert figure 2 here>

Fig 2. In the style of Latour, 1993, p.11

The general point then seems to be something like the need to keep notions of 'conscious and clear organization' simultaneously in play with the 'dark side of the system'. Unraveling the system into a network can then analytically perform the breaking up of the idea of management. Summing the network back up into positions and functions does the reverse work of showing how complex sets of relations become simplified as apparent hierarchies and dichotomies. But at what point is the transition from one to the other accomplished? Travelling from one direction, we often go from one set of terms to another and so on, until the swarm of relations that emerges might just as well be called a network. Starting from the reverse direction, the network starts to implacably stack up so many loops and displacements that at a certain moment it is, of course, a system for all formal purposes.

What is interesting about both directions of travel is that the transition point comes not in the middle of the account, but right at the end, as a sort of penultimate turning around on the analysis. Deleuze \& Guattari (1983) once used the phrase 'conjunctive synthesis' to describe these kinds of naming practices - 'Ah, so it's this after all!'. They compared them to the burp of the freshly fed infant, a sign that indicates both a completion and transformative recapitulation of the process. So the system really was a network, after all was said and done. How strange, that all these relations and translations came to act as system, at the end of the day. The conjunctive synthesis is a kind of heavily scaffolded gestalt switch. In this respect, consider an image with which we are all familiar:

<insert Figure 3 here>

\section{(Fig 3. E. Hill, Moja żona i moja teściowa, 1915, Creative Commons)}

The viewer starts with one picture, the thing they see first, either the old woman facing towards bottom left, or the young woman turned away towards the frame. To nudge the viewer from one image to the other, we typically point out key features. Look there, where the nose becomes a chin, the eye morphs into an ear, the mouth changes to a necklace. But despite this gradual preparatory work, the switch happens all at once: 'Ah, so it's....'. Critical work takes this same format. Extensive thick description prepares the ground for the penultimate 'reveal' where the organization changes, all at once, in its entirety (see Knox et al, 2015). To use the classical language of illusion popularized by Christopher Nolan's film 
The Prestige, the analytic breaking up management involves a) the pledge that there is a 'dark side' to the system; b) the turn of thick description of relations and c) the prestige of the eventual gestalt switch from system to network.

But let us return for a moment to the two long quotes by Serres and Latour. A historical account of Actor-Network Theory might see the latter quote as the answer to the former. If we wish to cede the identification of two interdependent modes of ordering to Serres, then Latour's efforts to demonstrate the necessity of thinking both the analytical and the constitutional places where these modes cross (and double-cross) one another gives us our contemporary critical position. However, what if the dark side of the system is just not its inversion into a network or the effect of the gestalt switch from one mode to another? What if there is another kind of emergent space, a third term between system and network? It would be difficult to perceive this kind of space, if it has any kind of existence at all, because it lies in the hinge or crossing space between the two terms. This would be a dark space not simply in terms or a reversal of what is usually perceived, but dark as a negation of perception entirely.

The critique of management typically proceeds through continuous inversion, upending the organization this way and that to show how the two modes of 'light' and 'dark' depend upon one another. It is this sense that critique can appear to be parasitic, in the common use of the term, since it attaches itself externally to its objects of study, and creates its own value through the demonstration of how to invert the image, now light, now dark. However, in doing so it leaves management pretty much untouched, since it does little to disrupt or destabilize the internal relation of 'light' and 'dark' to one another. What is required is not less parasitism, but a more effective form - an endoparasitic criticality that is capable of entering into management and organization practices, rather than just attaching to them externally, and seeking out the third spaces where reversals in modes of ordering occur. It is here, in interstitial spaces that emerge in the alternation between modes of ordering that a genuine disruption or breaking apart might not only located, but also fostered and encouraged.

II

China Miéville's The City \& The City begins as detective story. The story opens in the central European sounding city of Besźel with Tyador Borlú of the Extreme Crime Squad summoned to investigate a murder scene in a run down former industrial area. The narrative proceeds as one would expect, with the sifting of clues and the assigning of responsibilities, right until Borlú is preparing to depart:

Rackhaus said something that I ignored. As I turned, I saw past the edges of the estate to the end of GunterStrász, between the dirty brick buildings. Trash moved in the wind. It might be anywhere. An elderly woman was walking slowly away from me in a shambling sway. She turned her head and looked at me. I was struck by her motion, and I met her eyes. I wondered if she wanted to tell me something. In my glance I took in her clothes, her way of walking, of holding herself, and looking. With a hard start, I realized that she was not on GunterStrász at all, and that I should 
not have seen her. Immediately and flustered I looked away, and she did the same with the same speed. I raised my head, towards an aircraft on its final descent. When after some seconds I looked back up, unnoticing the old woman stepping heavily away, I looked carefully instead of at her in the foreign street at the facades of the nearby and local GunterStrász, that depressed zone. (2009: 14)

The woman who Borlú sees is not, in fact, in the same city. She is a resident of Ul Qoma, an entirely distinct city which, through a speculative fictional device which is never completely unpacked in the novel, is situated in the same topographical space as Besźel. Many parts of these two cities, superimposed on one another, appear to exist in something like parallel dimensions. One can be at the same geographical point in either city, and, for practical purposes, be unconcerned with the existence of the other. Both cities have their own particular history, culture, architecture and political system. They exist as something like independent sovereign states, with their own separate sets of international relations to the rest of the world:

If someone needed to go to a house physically next door to their own but in a neighbouring city, it was in different road in an unfriendly power. That is what foreigners rarely understood. A Besź dweller cannot walk a few paces next door into an alter house without breach. But pass through Copula Hall and she or he might leave Besźel, and at the end of the hall come back to exactly (corporeally) where they had just been, but in another country, a tourist, a marveling visitor, to a street that shared the latitude-longitude of their own address, a street they had never visited before, whose architecture they had always unseen, to the Ul Qoman house sitting next to and a whole city away from their own building, unvisible there now they had come through, all the way across the breach, back home. (p. 86)

Despite their topographical superimposition, there is only one place - a crossing/border point known as 'Copula Hall' - where it is permitted for residents of Besźel and Ul Qoma to cross to the other city. Proximal points, such as neighbouring house, are, in fact, topologically or relationally distant to one another, since they can only be legally reached through an enormous physical and political detour. But there are also other points of contact where the two cities meet in more ambiguous ways, known as 'cross-hatched' areas. Here the boundaries between Besźel and Ul Qoma can appear porous. Roads are shared, buildings appear to shade into another. Passersby in each city mingle together, present in the same topographical space, but oriented towards their own sets of distinct topological socio-material relations and coordinates.

The central device of the novel concerns the separation of the two cities. Besźel and Ul Qoma are not merely distinct political entities, they are policed as divorced phenomenological realities. Residents of each city must maintain the boundaries between the two states by systematically ignoring or 'unseeing' the other:

When an Ul Qoman stumbles into a Besź, each in their own city; if Ul Qoman's dog runs up an sniffs a Besź passerby; a window broken in Ul 
Qoma that leaves glass in the path of the Besź pedestrians - in all the cases the Besź (or Ul Qomans, in the converse circumstances) avoid the foreign difficulty as best they can without acknowledging it. Touch if they must, though not is better. Such polite stoic unsensing is the form for dealing with protubs - that is the Besź for those protuberances from the other city. (p. 80)

The boundaries between the city are maintained by an individualized disciplining of the senses that becomes almost a reflex action. Citizens are inculcated into a regime of 'un-seeing', deliberately not noticing, along with 'unhearing', 'un-smelling' and so on. This requires considerable cultural and practical skill, particularly around shared roads, where drivers must orient to one another at the fringes of their perception, simultaneously ignoring and taking care of the position of their 'topol-gangers' in the other city. The extensive training of the senses is assisted by an agreed upon maintenance of distinct semiotic codes around speech, movement, dress and architecture for the two cities. Knowledge of these codes allows citizens to selectively focus their attention on their own city, backgrounding that of the other that impinges in a shadowy way on their fringes of their senses.

Each city maintains its own juridical system. Whilst there are political points of contact between the two cities, in order to resolve disputes around shared resources, there is a separate mechanism that ensures compliance with 'unseeing'. Unauthorized crossing over between the two cities, through acknowledging 'topol-gangers', is known as 'breach'. Breach is the name for both the act itself of violating the phenomenological and legal separation of the two cities and the mysterious power that enforces this separation:

'Breach'. 'Breach'. I thought it was a shocked declaration by those who had witnessed the crime. But unclear figures emerged where there had been no purposeful instants before, only the milling of no ones, the aimless and the confused, and those suddenly appeared newcomers with faces so motionless I hardly recognized them as faces were saying the word. It was a statement of both crime and identity. (p.285-6)

The relationship between the sovereign powers of the two cities and that of this strange third power that underwrites their separation is the principle thread to the story. In some histories, Besźel and Ul Quoma were once a single city, which at a certain point divided. The identity and fate of this autochthonic origin of the two cities - known as Orciny - has passed into myths that treated as either fairy stories for children or dangerous subversions. In some versions of the myth, Orciny did not fragment at all, but continues to exist as a hidden third space between the cities. Perhaps Orciny is actually the name through which Breach refers to itself.

Two cities, then, functioning as distinct systems, with their own infrastructures and organizational forms, sat grosstopically in the same space. Between the two, a clear boundary which allows communication only through very specific institutional and diplomatically processes. Maintenance of the boundary ceded to a third, shadowy force which seems to exist as a third place that is both 
everywhere - since the powers of observation and intervention that Breach enact appear almost limitless - and nowhere:

THE BREACH WAS NOTHING. It is nothing. This is a commonplace; this is simple stuff. The Breach has no embassies, no army, no sites to see. The Breach has no currency. If you commit it it will envelop you. Breach is a void full of angry police. This trail that led and led again to Orciny suggested systematic transgression, secret para-rules, a parasite city where there should be nothing but nothing, nothing but Breach. (p.297)

Breach is parasitic on Besźel and Ul Quoma. It has no sovereign powers of its own beyond its role in serving as the conditions of the formation of the two cities as political entities. Breach is nothing, is grounded in nothing, and has nothing to trade or even communicate beyond its own parasitic existence. This becomes clear late in the novel, when it is revealed that the 'angry police' who are recruited into Breach are effectively 'press-ganged' from the two cities. The transgressors become the next generation of border guards. Or as Breach put it simply: do what you will in your own cities, but if you breach then you're ours. The boundary is nothing and nowhere, until it is revealed through the act of breach. And then it envelops you...

What begins as a detective story then ends up a parable about how the coexistence of two vastly different spaces is maintained by the withdrawing of the procedures for their maintenance into an in-sensible third space. The idea of a secret power - Orciny - proves to be a ruse. It is a device to attract critique and dissent and misdirect it across the boundary (the clues to find Orciny are always elsewhere, in the other city from where one is searching). Breach itself is not a secret, since it is always acknowledged. But its power to both disrupt and maintain, to break and reconnect, comes from its displacement. Breach alone provides the space for a deconstruction of the grosstopic arrangement of the two cities, whilst also providing the means for this ongoing continuation. As Tyador Borlú discovers, the only space that provides for a critique of Besźel and Ul Quoma is, literally, the nowhere space that exists between them.

\section{III}

Forensic psychiatric care is a practice for the management of persons with mental health issues who have either been accused of or convicted of criminal offences that exists outside of the penal system. It is usually embedded within the general hospital system, maintained in the UK through the National Health Service (NHS), rather than within prisons. There are three levels of secure care high, medium, low - that are implemented in different sites. The highest level of care is reserved for service users who have typically been involved in a significant crime (or 'index offence') for which they are deemed either not responsible due to their mental health at the time, or unsuited to the prison system by virtue of their ongoing mental health issues. Patients at these sites will typically be in the system for decades rather than years. Medium-secure units are normally attached to hospitals, and act as sites where service users 'transition' from the prison or legal system into psychiatric care, or downwards from high-security care. Time spent in medium-secure care can vary from around 3-15 years, depending on the rate of progress made in stabilizing the 
patient's mental health. Service-users in both high and medium secure care are formally detained under a Part 3 section of the Mental Health Act (i.e.

'sectioned'), and are not at liberty to leave until either a Mental Health Tribune or the Secretary of State (for convicted prisoners) deems it appropriate. Low secure care takes place in hostels and sheltered accommodation within the community, and represents the penultimate point in the rehabilitation process.

The espoused logic of forensic mental health care is to enable the recovery of the patient to a sufficient level where they can either be returned back to prison or released into the community to engage with outpatient mental health services. The guiding principle that governs decisions around transitions through secure care is the assessment of the risk to self and risk to others on the part of the patient. Note that recovery is not equivalent to cure - once a diagnosis of a mental health condition has been made, the goal is to stabilize symptoms and behaviour to a degree where they can be self-managed (a common, albeit contestable, comparison is with diabetes). Secure care is very expensive to provide, especially in cases where there are complex needs, and there are financial and political pressures on service provision, leading to increasing reliance within the NHS upon out-sourced care with Charity and commercial providers.

The following observations are based on empirical work in two medium-secure units located in a large city in the South of England (Brown et al, 2014; Reavey et al, n.d.). Medium-secure forensic psychiatric units look like prisons, and if they were not usually placed on hospital grounds you would easily mistake them as such. The walls and entry points to the unit are secure and designed to prevent unauthorized entry and exit. There are high walls, locked doors, airlock style entry systems, cameras and continuous staffing presence. Who and what goes in and out is closely monitored. This is ritualized in the practices of control and surveillance, including routine searches of wards and patient's bedrooms. Entering the unit can take some time and is subject to the working patterns of nursing staff, many of whom are managing multiple and sometimes unpredictable demands on their time.

Staff typically characterize their day-to-day activities in terms of the 'calmness' of the ward on which they are working (a medium-secure unit usually has around 6-8 individual wards which are divided by factors such gender, length of stay and diagnosis). A 'calm ward' is a general atmosphere defined by minimal agitation on the part of the patients and reasonably unproblematic relationships between patients and staff. Being able to sense changes in the atmosphere is a key skill that nursing staff develop, which draws upon sensitivity to a range of visual and auditory cues in the space of the ward (Kanyeredzi et al, n.d.). There are routine events that threaten the atmosphere, such as the admission of new patients transferring from prison and unsuccessful Mental Health Tribunals, along with daily activities such as mealtimes and arguments over the selection of channels on the communal television. Probably the single greatest challenge is the sheer lack of activities available to patients. Outside of semi-regular group sessions aimed at developing basic life skills, there is little by way of either therapeutic or recreation activities. Watching television communally or laying down in a bedroom comprises the major part of a patient's day. 
The maintenance of a calm atmosphere on the ward, accomplished through the stabilization of patient behaviour, is then the primary practical goal sought by nursing staff. Whilst work on ward exposes staff to some dangers, such as being injured whilst intervening in patient's behaviour, the primary risk is to the institution. Losing control over some aspect of the ward environment (e.g. the preparation of hot drinks) or of the ward itself is the most significant threat. There is then a disjunction between the espoused logic of the practice, which seeks patient recovery through minimization of risk to self and others, and the practical logic of running a ward, which aims at patient stabilization as a means of reducing risk to the institution.

This divergence in logics can be illustrated in a number of ways. A ward is composed of a number of communal spaces, such as a central living area and a dining space, along with individual patient bedrooms and small meeting rooms (where appointments with consultant psychiatrists and tribunals are held). The nurses' station is usually located at a central point, with clear lines of sight around the majority of the ward. Since nurses need access to records, computers and some medical supplies, part of the station will be within a lockable room, with large glass windows. On one occasion we were sat in the locked room waiting for an appointment. A number of nurses were present, updating the daily electronic records detailing the condition of patients on the ward. This was being done by cutting and pasting details from the previous day, and, where needed, making adjustments to the text. Whilst this was being done, a patient was repeatedly knocking on the door, requesting that staff give him access to an ironing board. He was initially ignored by the nurses, then discouraged. The door remained locked. We were told that responding to minor patient requests whilst within the room was counter-productive, since it simply encouraged more such requests, disrupting the flow of activities. The orderly management of interactions on the ward clearly took precedent over monitoring individual needs.

A recent major complicating factor has been the imposition of a ban of smoking in both the inside and outside spaces in mental health units. Before the ban, it was common to observe patients clustering around the nurses' station, waiting for a member of staff to accompany them to an outside space and provide them with a lighter to smoke. In many wards, staff developed informal work practices to routinize these requests through rotas or, even, we have been told, through a kind of 'production line' where queues of patients received a cigarette from one member of staff, which was lit by another, as they passed through the door to a garden space, before processing back inside. Clearly these practices are at odds with the health logic that underpins the goal of recovery. But they are consonant with the logic of maintaining a calm ward. Significant numbers of service users are smokers, who have large amounts of time with very little to do, and are legally prevented from doing the one thing that might alleviate their frustration. The informal management of smoking reduces collective aggravation between staff and patients (Matthews et al, 2005).

Another instance of the tension between health agendas and staff routines is around kitchen spaces. For many service users, food is a major source of complaint. Meals are normally prepared outside the unit and are served 
according to strict menus chosen well in advance (this is done in part to avoid arguments over choice and portion size when they are served). Not being able to choose either what or when to eat is a continuous source of patient dissatisfaction. This is often recognized on wards where patients are close to staged discharge, and where some facilities may be made available for selected patients to do some cooking for themselves. In one newly built unit, we observed that the design of wards included the provision of a small kitchen. However, when we asked patients how regularly they were able to make use of kitchens, we were told that they were rarely used (this was also apparent from their spotless condition). Cooking activities require a ratio of two staff members for every patient, ostensively because of the risks involved in access to kitchen equipment and cutlery. Losing the equivalent of four person hours of staff time for every session with two patients clearly detracts from the overall management of the ward. So for the majority of the time, the kitchens remain locked and patients can only stare in through the windows and imagine what they might have been able to do.

The vast majority of wards are separated by patient gender. One of the rationales for this separation is the management of the risk of (hetero) sexual predation and exploitation amongst 'vulnerable' adults (Brown \& Reavey, 2016). Personal and sexual relationships, whatever their orientation, are broadly discouraged and normally subject to sanction within medium-secure units. Nevertheless patients do engage in clandestine sexual activity with one another and form personal relationships. Staff are aware of these possibilities, and the ways in which particular ward designs may afford 'blindspots', or of the opportunities that leave to visit the hospital grounds may afford (e.g. sexual liaisons in public toilets). Whilst the institution itself cannot tolerate patient sexuality, because it is indexed to risk to self-and-others (Brown et al, 2014), nursing staff seem to tolerate moderate sexual banter and accept that patients progressing towards transition to the community are likely to engage in sexual activity (particularly on community visits). What is formally seen as threatening to recovery is treated as practically assisting stabilization as the patient moves through the forensic psychiatric system.

Medium-secure units are then composed of (at least) two competing orders of reason, an espoused logic and a practical logic. For the most part, these co-exist and orient towards one another without having to declare their

incommensurability. However, there are moments when the two orders seem to collapse into one another and a third kind of space briefly emerges. For example, modern ward designs place considerable emphasis on sightlines. Placing an open nurses station at the very centre of the ward, and then arranging communal spaces immediately around the station, with bedrooms and other rooms as 'spokes' radiating out from the centre, means that staff who sit at the station can maintain surveillance over the greater part of the ward space. However, as mentioned previously, this apparent control over the ward exposes staff to continuous requests from patients, and as a consequence, nursing staff often place themselves in the locked office part of the station. Moreover, the control provided by the central location is reversible. As one staff member observed, it requires only one agitated patient, armed with a pen, to take a hostage at the 
nurses station, for control to be ceded control, since the patient would now be able to take advantage of all the sightlines.

The formal and the informal logics that are in play around the nurses station (e.g. the control of sightlines and the maintenance of work routines) are suspended or breached by the possibility of hostage taking. Power shifts away, if only temporarily, from both the control mechanisms of the institution and the informal management strategies of nursing staff. It becomes difficult to discern the difference between risk to self-and-others and risk to the institution, which merge into a critical situation. Admittedly, this kind of event is comparatively rare. It is 'nothing' compared to the routine dramas of life on the ward and yet it is 'everything' in respect to the coherency of the normal logics in play, since it threatens their separation. Why focus on recovery, when there is such an enormous risk? Why try to work towards a 'calm' atmosphere, when it can go so catastrophically wrong? The breach of hostage taking is like a shadow that reminds staff of just why it is that the formal and the informal cannot acknowledge one another, since to do so would make their particular versions of care mutually unworkable. Anticipation of this breach is then a major dynamic within the unit. Often unspoken, it is third order of reason all to itself that haunts and divides the other two. It represents both the transgression of the dual ordering of the ward, and the basis for their ongoing regulation. It is some sense both the 'real' site of management - the space that must be continuously anticipated within everyday decisions made on the ward - and the basis on which espoused management is disrupted. Because breach, the moment where the calmness of the ward collapses, really is the moment where the logics of risk and containment become undone.

IV

\section{Definition 1:}

A 'station' or 'position' is a formally recognized site within an organization to which powers-to-act, responsibilities and accountability are accorded

A 'relation' is a mode of connection between two stations that is characterized by a reciprocal movement of acting and being acted upon

\section{Proposition:}

Organizations are composed of stations and relations. Stations can be purely discursive objects (i.e. 'vision statement'), material arrangements (i.e. 'head office') or, in the majority of cases, a mixture of both (i.e. 'management'). Relations define stations through their continuous communication with one another. Stations present themselves as the 'summing up' of the relations through which they persist.

\section{Demonstration:}

No station can exist outside of a network of relationships to other stations, since such an existence would have no modes of connection and communication with other stations. Entering into a network subjects a given station to reciprocal definition and shaping by other stations. In this way, no station can ever acquire 
an irreversible status, since it becomes dependent on the network of relations through which it acts and is acted upon.

\section{Corollary:}

If power is considered as power-to-act, any extension of the powers of a given station implies the growth of a network, which exposes the station to a greater range of reciprocal actions and increases its dependence on the network as a whole.

\section{Definition 2:}

'Formal organization' is primarily concerned with mapping the distribution of stations relative to one another.

'Informal organization' concerns the multiplicity of relations that obtain between these stations and how they appear to the persons who occupy stations from their respective positions.

\section{Proposition:}

The representation of an organization differs entirely depending on whether it is stations or relations that are being mapped. Stations are considered to have an independent existence, by rights, outside of the relations in which they are embedded. Relations are, in practice, corrosive of the clear definition of stations. If the formal or espoused logic of an organization is presented in terms of the distribution of stations this will inevitably be insensitive to the actual forms of connectivity and communication through which an organization operates (i.e. how things actually get done). If the informal or practical logic of an organization is presented merely in terms of relations then it will fail to account for asymmetries in the distribution of rights and accountabilities. Moreover, any account of an organization that does not alternate between stations and relations will be unable to articulate their interdependency.

\section{Demonstration:}

Stations are defined by reference to an external process or agency, which accords them rights and formal powers in principle. This lends them the appearance of stability and completeness, since the act of definition does not necessarily depend on any internal process. But these external sources of definition do not by rights secure the practical means to act, creating a gap between status and effectivity. Relations are defined through internal processes within the network and are, in practice, subject to continuous, ongoing revision. Because they are not dependent on external authority, relations may appear to be both weak (in that they cannot necessarily draw upon outside sources of legitimation) and strong (in that they have a relative autonomy from outside forces). From this it is clear that any adequate representation of an organization will have to be concerned with both the external, de jure constitution of stations, and the internal, de facto working out of relations.

\section{Scholium:}


The difficulty of containing descriptions of both stations and relations within a single account pushes the analyst towards dualism - formal vs informal; light vs dark; system vs network and so on. Moreover this dualism is a resource for organizational members themselves, who may readily alternate between accounts of 'what should happen' and 'what actually happens'. This renders the connections between stations and relations ever more mysterious, as though they become one at various points through some kind of interactional alchemy. The task of the analyst then becomes that of stopping this alternation through seeking the moments when it breaks down or is acknowledged as temporarily impossible, through finding the space that serves as the catalyst.

\section{Definition 3:}

'Parasitism' is the interception of a relation between two stations. It diverts what flows through one relation into a new direction. A parasite typically does not have the status of a 'station', although some stations may deliberately seek to act in a parasitic mode.

A 'parasitic cascade' occurs when one act of interception is interrupted by a further act. This creates a vulnerability that attracts further interceptions without a clear stopping rule.

'Value' is the extraction from or redirection of a flow between two stations into a new direction.

\section{Proposition:}

Parasitism is an inevitable feature of the relational arrangement of stations. Because stations depend upon one another, they must risk a mode of connection or communication that passes through a medium which is not under their complete control (i.e. it is outside the definition of the station). Any given flow of information, materials, affect or discourse - is a potential source of value to an external agent who is capable of grasping a portion of that flow, or redirecting some of it. Ergo: management is constituted through its own breaking. In some cases, 'leaders' may seek to act in a deliberately disruptive manner that renders them as equivalent to parasites, but with considerably less potential for novelty.

\section{Demonstration:}

Parasites extract value from the flows between stations and appear to offer nothing in return. Parasites differ considerably in scale to the stations whose relations they parasitise. As such, they may appear to be invisible, or at least 'unseeable', to stations. Parasites exploit vulnerabilities in the relational structure of networks. There can be no network without the possibility of parasitism, since the power of a network depends upon its spread, which multiplies the points of attack. However, the enactment of parasitism creates a new relation, which multiplies the number of stations involved. The parasitic relation may, in turn, be parasitized by other parasites, which are able to discern novel sources of value in the extractive process. This process can be multiplied exponentially since, as a process internal to a network, there is in principle no external stopping rule to parasitism. 


\section{Corollary:}

We live with our parasites. The only sure defence against parasitism is to retreat from relations, which is, in effect, to break all connections to others. In a purely relational sense, power names the spread of networks, which is simultaneously the multiplication of the possibilities for parasitism. However, given the acknowledgement of parasitism within a given field of power, the position of maximum strength comes from the anticipation of parasitic cascades and the strategic positioning as the 'last in line' of all the parasites. Hence, organizations both anticipate the inevitability of parasitism and develop the means to parasitise their own parasites in such a way that they can manage and contain parasitic cascades.

\section{Definition 4:}

'Copula' are the acknowledged points of passage between two or more sociomaterial logics.

The 'dark side of the system' properly names a place that it is in-between the formal and informal, the espoused and the practical, the system and the network.

'Breach space' is the emergent 'third space' that occurs when a parasitic cascade interrupts the arrangement of stations and relations.

\section{Proposition:}

Organizations are composed of stations and relations, which are implicated in different modes of reason. Stations are distributed with reference to external authorities, whilst relations unfold according to an internal, emergent logic. The incommensurability of the logics of stations and relations is widely acknowledged within organizations, as is the need to alternate between logics on occasion. However, this acknowledgement masks a broader interdependency. This implies the existence of a 'third space' where both the two logics combine, mix together, or become difficult to separate, in a way that would otherwise be regarded as impossible. This occurs during moments of 'breach', where the divergence of the two logics is attenuated and collapsed. Such a space occurs during moments of interruption to both the formal and the informal sides of organization, where both become simultaneously paralysed and catalyzed to a novel mode of functioning.

\section{Demonstration:}

Despite their interdependency, it is difficult to describe the distribution of stations and the patterning of relations in a single account of organizational life. Members can readily acknowledge that 'but of course' it is necessary at times for there to be an alternation between logics. Whilst such copula points are typically not advocated within the logic itself, they are rarely treated as 'secrets' or entirely sanctionable matters. The 'dark side' of an organization is then neither a hidden internal structure, nor an as-yet-unmasked set of external relations, but rather a penumbral space at the limits of perception in which the two logics cohere. Ordinarily, this space is nowhere - it is not part of the mundane day-today functioning of the organization. But its existence is a necessary moment of 
concrescence where the co-existence of incompatible orders of reason is accomplished. It is during periods of crisis, when the relations between stations are parasitized, that the space temporarily emerges and is recognized as such. Parasitic cascades interrupt and disrupt the separation of the formal and the informal and create a superordinate threat to their mutual functioning.

\section{Definition 5:}

A 'parasite' is a form of agency that is initially smaller in scale and capacity-to-act than stations. Parasites do not typically exhaust or destroy their hosts. Their destructive power is often matched by their creative power.

\section{Proposition:}

Parasites are disruptive of the usual functioning of systems. They exploit vulnerabilities by finding ways of diverting small amounts of flows to create value. Parasites cannot produce order for themselves, but require there to be a prior form of order onto which they can latch. Organizations very rarely collapse under the weight of parasites, except under comparatively rare circumstances of uncontrollable parasitic cascades. Here, parasites can become sufficiently engorged as to overcome their host, but in doing so they merely become a vector of transmission for other, nimbler parasites. Although parasites appear to take without giving, because they create new forms of relationality, and force organizations to confront the incommensurability of stations and relations, they serve as catalysts for change.

\section{Demonstration:}

All organizations tolerate a certain degree of 'leakiness', in strategic, financial or material terms. The flows that compose an organization are always vulnerable to extraction by a third party. If this exploitation remains at a sufficiently small scale, the organization is unlikely to see the outright rejection of the parasite as a pressing priority. However, the possibility of the escalation of parasitism is a significant concern, and for this reason apparently minor events may become unduly important to the organization because of the anxiety around scaling up. This anticipation may have value to the organization, because it acts as driver for reviewing its own practices and relationships that lie outside its own networks. In the rare cases where the parasite actually takes the place of the organization, it rapidly becomes the target for immediate further parasitism.

\section{Scholium:}

Is a parasite a station or a relation? Probably neither, although some stations act in 'parasitic' manner, and some instances of parasitism become sufficient intense as to give rise to what appears to be a new station. Is parasitism a 'good' or 'bad' thing? The question is meaningless in itself. Parasitism inevitably happens and constitutes a threat and a cost to existing stations and relations, but draws out novel responses that can transform stations and multiply relations. Is the analyst a 'parasite'? On most occasions, probably not, since the analyst rarely 'breaks' or 'paralyses' any part of the organization, but instead typically latches onto an existing vectors of parasitism and thereby 'parasitises the parasites'. 


\section{Definition 6:}

'Anticipation' is the imagining of possible futures based on the prolongation of the current logics in play and the potential challenges they may encounter.

'Anticipation of breach' is an unspeakable acknowledgement of the collapse of different orders of reason into one another in emergent, yet predictable, events.

\section{Proposition:}

Organizations are principally driven by the anticipation of breach. Although these moments are comparatively rare, they cast a long shadow over the organization. In breach, the organization is forced to confront the incommensurability between the logics in play, and the necessity of their separation. Breach is a moment of collapse and recognition. Since the ongoing functioning of the organization relies precisely on the 'unseeing' of divergences in logic, despite tolerance of their mutual co-existence, breach presents itself as the unraveling of the coherence of the incompatible. But in the same way that the recognition of the inevitability of parasitism has a productive value, so the unspoken acknowledgement of breach does the work of enabling the organization to maintain an active engagement with its own contradictions.

\section{Demonstration:}

A given order of reason within an organization is defined against a second order, whose projects and values can only be tolerated if they are amenable to translation. In this way, orders of reason may cohere in an organization because it appears that everyone is signed up to the same objectives, despite the fact that these goals have been significantly re-imagined and re-purposed across the competing logics. Rather than being actively repressed, the incommensurability between logics remains acknowledged. The possibility of breach represents a moment where the separation of orders may collapse, revealing their mutual foundation in a project which is incomprehensible if it were to be subject to a single definition. Thus, avoidance of breach is an act that both restates the importance of the separation of logics and tacitly affirms their dependence. The avoidance of breach is therefore a major driving force within the organization. This might be considered as a third, unrepresentable and unspeakable order of reason that underpins those that are overtly in play.

\section{Afterword}

Dark organizational theory is a descriptive practice rooted in an ethnographic sensibility that focuses on the divergence between different orders of reason in organizational and institutional settings. It treats incommensurability not as an obstacle, but rather as an inevitable, functional aspect of organizational life. As Deleuze \& Guattari (1983) once quipped 'things work because they don't work'. Coherence between diverse local orders of reason is a routine accomplishment. It does not come as a surprise to members that there are different agendas and modes of ordering in play that are not, in principle, easily resolved, and may in fact be seemingly at odds with the espoused purpose of the organization. Whilst there is intrinsic value, we would argue, in giving voice to 'stories from the 
frontline' of modern organizational life, doing so does not amount to a critical work of revealing what was supposedly 'hidden'. The contribution of dark organizational theory is instead to demonstrate that whilst competing sociomaterial logics can be generally acknowledged within an organization, their mutual co-existence requires a systematic work of 'unseeing' and 'unsensing' in everyday practice. This requires considerable 'affective labour' on the part of members who not only have to the face the challenge of enacting their own practice, but also ensuring that they 'disattend' as far as possible to the rival logics that are in play (even when these alternative logics are potentially compelling). The focus is then on the local, contingent work of producing coherence in organizational and institutional settings that teeter on the verge of continuous incoherence.

Despite their incommensurability, sociomaterial logics have forms of interdependency. Some are clearly marked as agreed points of passage or 'copula', but other broader forms of interdependence only fully emerge at moments of 'breach', where a reversibility and collapse between logics occurs. Breach is an interruption or 'parasitism' of established relations that cuts across the differences of logics. It creates a temporary and unstable 'third space' of mixture where a new form of order is extracted, to the cost of the organization. Breach presents as a threat to the whole range of local modes of ordering and compels a response that inverts their existing logics (although it often concludes with the transformation and reaffirmation of incommensurability). The central proposition of dark organization theory is that anticipation of breach, rather than the specific goals of 'formal' or 'informal' organizing, is of central significance for understanding why organizations do what they do.

Critique then needs to begin by becoming attuned to when and how breach is possible, learning from the affective practices of members who are skilled in its anticipation. The moments where management - in its broadest sense - faces its own breakdown become pivotal to understanding how the organization goes on. The analyst has to learn to think like the parasite, pursue a kind of 'parasite logic' (see Brown, 2013) in their investigations. Dark organizational theory is not a theory as such, although it can be presented as a set of conceptual propositions ${ }^{3}$. It does not lend itself to easy empirical application, although it enhances sensitivity to 'third spaces'. It does not bring us any closer to what management is 'actually' doing. But it suggests, with some plausibility, we want to claim, tell us what it is that managers and the managed glimpse for a moment when they forget what it is that are supposed to be looking at.

\section{References}

Brown, S.D. (2002). Michel Serres: Science, translation and the logic of the parasite. Theory, Culture \& Society 19(3): 1-27.

Brown, S.D. (2013). In praise of the parasite. Informática na Educação: Teoria e Prática 16(1): 83-100. 
Brown, S.D., P. Reavey, A. Kanyeredzi and R. Batty 2014. Transformations of self and sexuality: Psychologically modified experiences in the context of Forensic Mental health. Health 18(3): 240-260.

Brown, S.D. and P. Reavey (2016). Institutional forgetting/Forgetting institutions: Space and memory in secure forensic psychiatric care. In Institutions Inc, eds. E. Weik and P. Walgenbach. London: Palgrave, pp. 7-29.

Burrell, G. (1997). Pandemonium: Towards a retro-organization theory. London: Sage.

Cooper, R. (2016). Organization/Disorganization. In G. Burrell and M. Parker (eds) Robert Cooper: Collected works. London: Routledge.

Friedland, R., and R. Alford (1991). Bringing society back in: Symbols, practices Formatted: German (Germany) and institutional contradictions. In W.W. Powell and P.J. DiMaggio (Eds) The New Institutionalism in Organizational Analysis. Chicago, IL: Chicago University Press, pp.232-263.

Hanlon, G. (2015). The Dark Side of Management: A secret history of management theory. London: Routledge.

Kanyeredzi, A., S.D. Brown, L. McGrath, P. Reavey and I. Tucker (n.d.) Bangs and Clinks: Sensing the visual and sonic spaces of a re-designed forensic psychiatric unit. Under Submission

Knox, H., D.P. O’Doherty, T. Vurdubakis and C. Westrup (2015). Something happened: Spectres of organization/disorganization at the airport. Human Relations 68(6), 1001-1020.

Latour, B. (1993). We Have Never Been Modern. Cambridge, Mass: Harvard University Press.

Linstead, S., G. Maréchal, and R.W. Griffin (2014). Theorizing and researching the dark side of organization. Organization Studies 35(2): 165-188.

Matthews, L.S., B. Diaz, P. Bird, A. Cook, A.E. Stephenson, J.E. Kraus and B.B. Sheitman (2005) Implementing a smoking ban in an acute psychiatric admissions unit. Journal of Psychosocial Nursing, 43(11): 33-36.

Miéville, C. (2009). The City and the City. Basingstoke: Pan Macmillan.

Mol, A. (2008). The Logic of Care: Health and the problem of patient choice. London: Routledge.

Mol, A. and J. Law (eds) (2002) Complexities: Social studies of knowledge practices. Durham: Duke University Press.

Raufflet, E and A.J. Mills (2009). The Dark Side: Critical cases on the downside of business. Sheffield: Greenleaf. 
Reavey. P., S.D. Brown, A. Kanyeredzi, L. McGrath and I. Tucker (n.d.) Spectral subjectivities: Life space on a medium secure forensic psychiatric unit. Under Submission.

Serres, M. (1982). The Parasite. Baltimore: Johns Hopkins.

Thornton, P.H., W. Ocasio, and M. Lounsbury (2012). The Institutional Logics Perspective: A New Approach to Culture, Structure and Process. Oxford: Oxford University Press.

Tourish, D. (2013). The Dark Side of Transformational Leadership: A critical perspective. London: Routledge.

Ward, J. and R. McMurry (2015). The Dark Side of Emotional Labour. London: Routledge. London: Routledge.

\section{Acknowledgement}

We would like to thank the three anonymous referees and the section editors for their extraordinarily helpful comments and suggestions, many of which they will see directly reflected in the text.

\footnotetext{
${ }^{1}$ The term 'competing logics' refers here to the relationship between diverse modes of constituting sociomaterial 'objects' (e.g. 'good care', 'reduction of risk', 'recovery') and 'subjects' ('patient with self-insight', 'empathic carer') within a specific practice, along with the 'rationales' that emerge within the mode (see Mol, 2008). This differs from the notion of an 'institutional logic' as a higher-level belief system rendered operant within a practice by way of a focus on both the emergent nature of the mode of ordering, and the manner in which meaning emerges through the local and provisional concrescence of subject and objects (e.g. shifts in the funding of care result in ongoing transformations of what counts as recovery and effective doctoring/nursing).

2 The idea of 'the third' or 'thirdness' has a long history in the humanities and social sciences, including the postcolonial theory of Homi Bhabha (2004) and Edward Soja's (1996) 'thirdspace'. The common thread there is that of mixture and the confrontation with alterity. In the context of 'dark organization theory', thirdness refers to the anticipation and temporary emergence of a space where the incommensurability of logics becomes starkly apparent and can no longer be adequately managed. It is informed directly by Michel Serres' concern with the impossible origins of multiplicity - i.e. 'trampled multiplicity', 'clinamen', 'parasitic cascades' (see Brown, 2002).

3 The extent to which these propositions draw heavily on Serres (1982) should be readily apparent.
} 